\title{
Leak Detection in Pipe Networks Using Hybrid ANN Method
}

\author{
Uttam Roy ${ }^{1}$
}

Received: 24 February 2017 / Revised: 4 October 2017 / Accepted: 4 October 2017 / Published online: 17 October 2017

(C) Springer Nature Singapore Pte Ltd. 2017

\begin{abstract}
The idea of hydro informatics is applied for determining the assignment dilemma accompanied to water-based mechanism. This consideration applied prototype data as well as assertion for developing advantageous prototype for waterbased approach. Hydro informatics can also assist for hydraulics, hydrology, and mechanical engineering. Hydro informatics applies software-based artificial technology for determining these consequences accompanied to water-based approach. Hydro pipe network is placed underground as well as once constructed, it is difficult to identify the status of ducts when excrete or crack occurs. Accordingly, post activity is frequently postponed later the error arises. Hence, the consistent error activity approach of water channel mesh is mandated to control the coincidence as well as lower the depletion. In this application, we compose online error identification approach of water channel network utilizing details of pipes similar as flow rate. The ephemeral prototype explaining water flow in pipelines is showed along with depicted utilizing MATLAB. The defect locations for waste or crack can be illustrated as discharge or pressure information's when the error combined. Errors identified by applying of FFT and DWT algorithms; additionally, it was contrasted for achieving favorable error breakthrough outcome.
\end{abstract}

Keywords Leak point localization · FFT (first Fourier transform) $\cdot$ Pipeline monitoring $\cdot$ Artificial neural networks

Uttam Roy

uttam_ju31@yahoo.co.in

1 School of Hydro-Informatics Engineering, National Institute of Technology, Agartala Barjala, Jirania, Agartala, Tripura West 799046, India

\author{
Nomenclature \\ UST Underground storage tank \\ FDD Fault detection and diagnosis \\ KF Kalman filter \\ EKF Extended Kalman filter \\ PDE Partial differential equation \\ ODE Ordinary differential equation \\ PF Particle filter \\ STF Strong tracking filter \\ FFNN Feed-forward neural network \\ DPT Differential pressure transducer \\ ADC Analog digital conversion \\ DWT Differential wavelet transform
}

\section{Introduction}

Pipelines considered as a safe means of medium to transfer fluids from one position to another. In practical applications, pipes function under many difficult conditions [1] The application of hydro informatics involved in the field of hydrosciences and engineering [2]. The rapid advancement and success of hydro informatics is allied with its wide range of applications [3]. This paper is focused on modern advances and highlights practical inference of hydro informatics technologies [4]. The practical use at Delft Hydraulics was demonstrated and presented [5, 6]. The uniform hydraulic system consists of pumps, valves, in-line instrumentation, and pipeline which transport fluid through pumping process. The pipeline can produce indicative signals which are analogous to leakage signal under standard operation. Therefore, designing a costeffective and reliable leak detection system is quite needful for products pipelines [7-9]. A guideline has been build up to assess the performance of leak detection systems to check the veracity of pipelines in USTs [10]. It affects leak detection 
systems attached to the pipeline and related with the loss of fluids through the pipeline under pressure. However, the system is not unique to predict enumerated leak rate [11].

In last two decades, leakage detection method has elevated as a trivial research area due to its wide application [12]. Fault detection and diagnosis (FDD) is an analytical model-based widely used approach where different filters (KF, STF, and EKF) are used. The evaluation capability of the particle filter (PF) was first introduced in FDD approach [13]. Random walk noise approach introduced to estimate the fault in augmented states which shows lesser accuracy of the method than PF. By this approach, fault detection can be done with accuracy and less time but without prediction of leak size [14].There are abundant methods for leakage detection. Most simple and common method is physical inspection [15]. But this method can predict only leakages visible from outside not inside the pipe materials. Therefore internal leakage detection emerged as an important area of research and several computational approaches had been introduced to envision the work. The pressure transitory methods have been applied as very useful tool [13, 11]. Zhi Sun et al. [16] proposed a solution for transferring bulky quantity of fluids over long distance. His work proposed a low-cost and real-time leakage detection system for underground pipelines. It utilized different types of sensors that are positioned both inside and outside the underground pipelines which detected leak existence. The measurements of different types of the sensors in pipeline network intimated to the screening unit in real time by implementing an MI waveguide technique.

Andrew F. Colombo et al. [17] proposed leak detection methods (transient-type) with description of present and past work in specific domain. To provide a reasonable range of research activities and various methodologies, numerous publications were cited.

But the major drawback is lack of field work and verification of these techniques [6]. Kingsley E et al. [18] used Liapunov stability criteria for pipeline leak detection considering liquid phase of the fluid. Liapunov stability criteria were utilized in model for pipeline leak detection. The velocity and pressure profiles were generated and validated with industrial dataset. Eigenvalues ( $\lambda$ 's) were determined by stability matrix developed from the deviation model of velocity and pressure. For eigenvalue, values less than 1 , leak is detected and for the eigenvalues greater than 1 , surge in the pipeline was detected. In oil-transporting pipeline section, simulation profiles of eigenvalues of a pipeline network and that pressure deviations were more sensitive parameter for leak detection. For larger leak systems, volume deviations emerge to be fine pointer. In this study, both single and double leak situations in a pipeline system were investigated.

Ming Liu et al. [19] have proposed EKF and STF filters in his work. A nonlinear pipeline model is required for this method. Monte Carlo methods are useful to estimate status in nonlinear and non-Gaussian systems with nonlinearity. Parameter estimation techniques were widely used in FDD and have been applied to pipeline leak detection. For timevarying parameter assessment, the particle filter algorithm is not suitable. The variance of the artificial noise is adjusted adaptively using adaptive particle filter algorithm. This method can be implemented for leak detection of gas pipelines. $\mathrm{M}$ Taghvaei et al. [20] transient analysis is one of the credible approaches for leakage detection due to minimalism and sturdiness. The waves developed due to fluctuation of pressures considered for analysis. The delayed time period between propagating waves and its reflections were identified using cepstrum method. This approach simplified difficulties for finding small leakages inside the pipeline and proposed further application for complex pipe networks.

\section{Motivation for the Research}

The review of literature has explored the inclination of the research in the carrying out tests in pipeline leak detection method. Most of the review works has performed the trace analyzed in the frequency domain method which can improve the characteristics performance fluid flow in pipe networks. It is applied to identify specific pipeline facet in different conditions. However, mechanical researchers use traditional method for pipeline leak detection system. Leak detection methods can be classified into two categories: transient method and inverse method to determine the leak factors through modeled frequency responses, inverse resonance method is used. It is well known that the development of corrosion model of physical and mathematical model technique to research in detection properties in real-time environment. The model was designed to facilitate extraction of additional information about the process without carrying out repetitive experiments. A less number of researchers have contributed to derive such type of model for the leakage detection process; only some of the research paper consider the knowledge-based mathematical model is the impact of leakage properties in real time industry. From literature review, it is found that very limited research work conducted in similar field which encouraged and motivated for research.

\section{The Proposed Methodology}

The objective of the research is to find more accurate prediction technique for leakage detection by resolving existing shortcomings in the literary works. This work proposed novel optimization properties in real-time environment. Propose technique will be done in two stages namely: optimization algorithm and mathematical process for prediction of leakage rate. In this paper, proposed model depict the leakage process 
in an improved way for actual physical structures under the Cestrum analysis and immersion mathematical processes is used to control the diffusion of the leakage rate in the underwater. The model will provide the leakage processing information as well as predict the leakage rate output in an efficient way. The technique will have to investigate the deleterious effect of the leakage on the process of the reaction on the product quality. Hence, the technique recommends derivation of precise mathematical model to exactly cope up with practical data in the leakage process. In this work, pressure difference employed as predictor for leakage detection using a hybrid artificial neural network model. The performance is validated in epochs and best validation performance is 0.21854 at 0 epochs. The implementation of proposed approach to predict the performance of leak detection in pipeline MATLAB is used as working platform.

\section{Model of Pipeline-Mechanical Modeling}

Figure 1 shows proposed model for leak detection process. Artificial leakages were constructed at the pipe and equipment were fitted before and after leak point for collecting, monitoring, and analyzing datasets. Pressure differences were recorded with the help of DPT and ADC employed for signal monitoring and conversion. The entire leakage identification unit was built using several sub-units like pressure measurement unit, data collection unit, and data analysis unit.

\section{Proposed Technique for Modeling Leak Detection System with Mathematical Formulations}

The flow transition phenomena inside pipelines due to leakages are well defined with the help of conservation of mass and momentum equations depicted by Eqs. (1) and (2)

$$
\begin{aligned}
& \frac{\partial H}{\partial t}+V \frac{\partial H}{\partial x}+\frac{a^{2}}{g A} \frac{\partial Q}{\partial x}=0 \\
& \frac{1}{g A} \frac{\partial Q}{\partial t}+\frac{V}{g A} \frac{\partial Q}{\partial x}+\frac{\partial H}{\partial x}+\frac{f Q|Q|}{2 g D A^{2}}=0 .
\end{aligned}
$$

Equation (3) considers wave velocity for the fluid flowing through the pipe with leakages.

$$
a=\sqrt{\frac{\frac{k}{p}}{1+\frac{K}{E} \frac{D}{e} \varnothing}}
$$

Factors explained in Eq. (3) depend on boundary constraints the piping system. The PDE's indicates transient model and particular solution is used to transform it into ODEs [6].

\section{Leakage Boundary}

Equation (4) describes discharge magnitude for flow through leak point and discharge magnitude before and after the leak point is incorporated in Eq. (5).

$Q_{L}=C_{d} A_{0} \sqrt{2 g H_{L}}$

$Q_{u}-Q_{d}-Q_{L}=0$

Where $Q_{u}$ as well as $Q_{d}$ are the discharge value before and after the leak point, are, obtained by Eqs. (2) and (3) by $H_{L}$ substituting $H_{p} . \mathrm{H}_{\mathrm{L}}$ can be calculated by using Eqs. (2) into (3), and Eq. (4) into Eq. (5). $Q_{u}$ as well as $Q_{d}$ can then be acquired applying $H_{L}$.

\section{Practicing Neural Networks}

The improved utilization of artificial neural network (ANN) requires reformation of the respective feasible benefits of various networks and knowledge of the advanced processes. Network topologies and training algorithms are consistently evolving and neuro-hydrologist should preserve abreast of similar arrangements. There are multiple applications of neural networks in various domains. But selection of suitable topology, algorithm, architecture and periods are crucial for better practicing neural networks. Many activities may be programmed applying suitable changes to train algorithms and several judgments can be made by repeated run.

Further, practicing method depends on network topology and algorithm training methods applied. Reviews show that LS method or HT methods are mostly utilized. In LS-method, $x$ depicts inputs, i.e., slanted total of neuron along with $\mathrm{f}(x)$, outputs utilized frequently due to its consistency and sootiness for evaluation. It projects the productivities from intense, as well as it produces complex behavior of NN.

$f(x)=\frac{1}{1+e^{-x}}$

In FFNN structure, neurons follow single path: starts from a participation layer, then passes through single or many hidden layers and finally move to production layer. Many studies explicitly envisioned output layer in participation side to construct additionally auguries, exactly remarking FFNN carry only one advance pass to complete setup for every augury.

\section{Artificial Neural Network Modules}

ANN is applied as an optimization tool for prediction of leakage in pipelines. The proposed method utilizes feed forward 


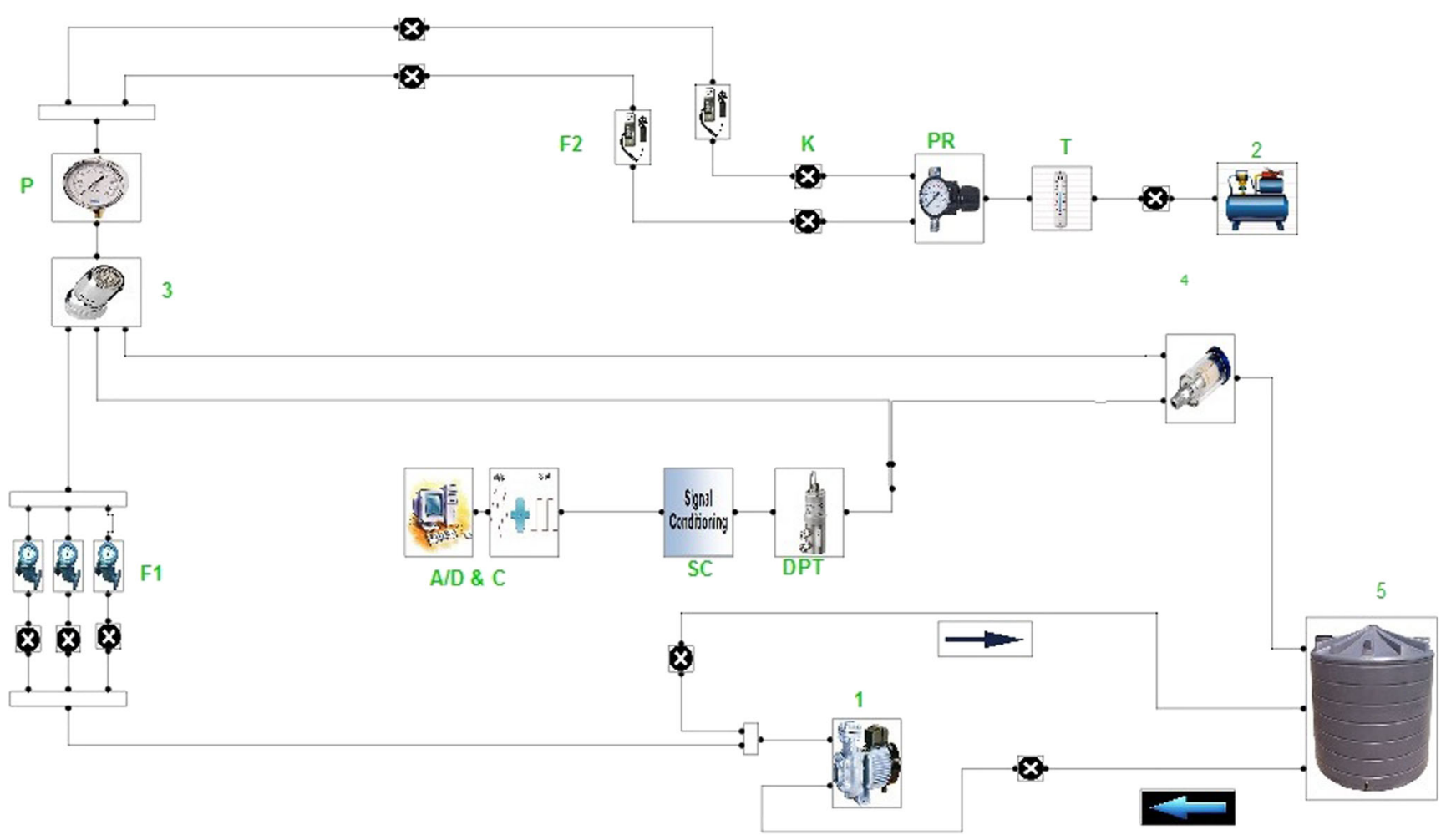

Fig. 1 Proposed model of leak detection process. Symbolic details: 1, pump set; 2, flow analyzer; 3 , pressure transducer; 4, solenoid (artificial leak point); 5, storage tank; $\mathrm{P}$, pressure gauge; $\mathrm{PR}$, pressure reducer; $\mathrm{F} 1$,

type neural network. The FFNN consists of an input layer, an output layer along with single or multiple hidden layers. The input layer consists of five inputs, i.e., $T_{1}, T_{2}, T_{3}, T_{4}, T_{5}$. ANN tasks as following manner: at every nodal point, $H_{i}$ in input layer there is signal $T_{i}$ as input of the network's multiplied by a weight value between the input layer and the hidden layer (Figs. 2, 3, 4, 5, and 6).

The training phase has three key steps, and it is associated with neural network performances. In this section, the artificial neural network (ANN) is proposed. The training of data set and testing the applied input data is accomplished flow valve (parallel); F2, flow valve (single); $\mathrm{K}$, stop valve; $\mathrm{T}$, transducer; DPT, differential pressure transducer; SC, signal controlling unit; $\mathrm{A} / \mathrm{D} \& \mathrm{C}$, analog/digital converter

by ANN approach. In this paper, we use the artificial neural network for finding the leak detection mechanism that combines with training input for the water-based approach of assembly leak detection systems. At the first leak, detection section is joined in a synchronization station from the dataset with the help of pre-processing process, which is discussed in previous section. Here, we utilize the feed forward type neural network, which has three major layers that are input layer, output layer, and one or more than one hidden layers in between the input layer and output layer. The training of neural network is complete with the help of

Fig. 2 The structure of ANN for proposed method

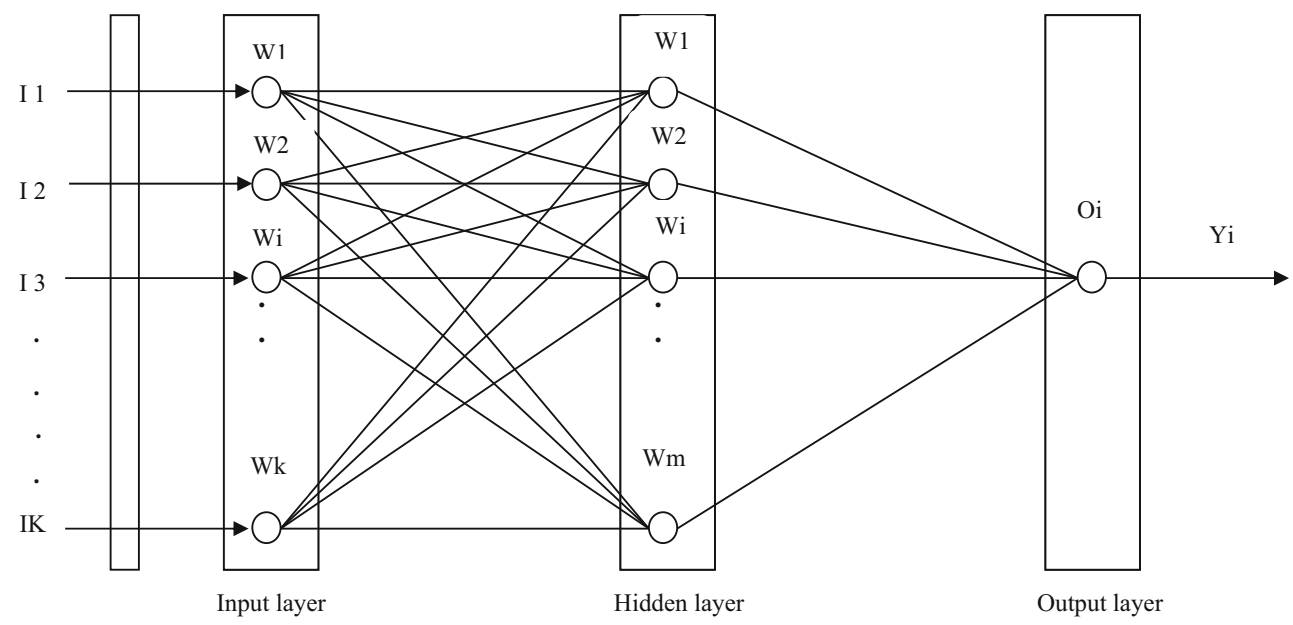


Fig. 3 ANN output target
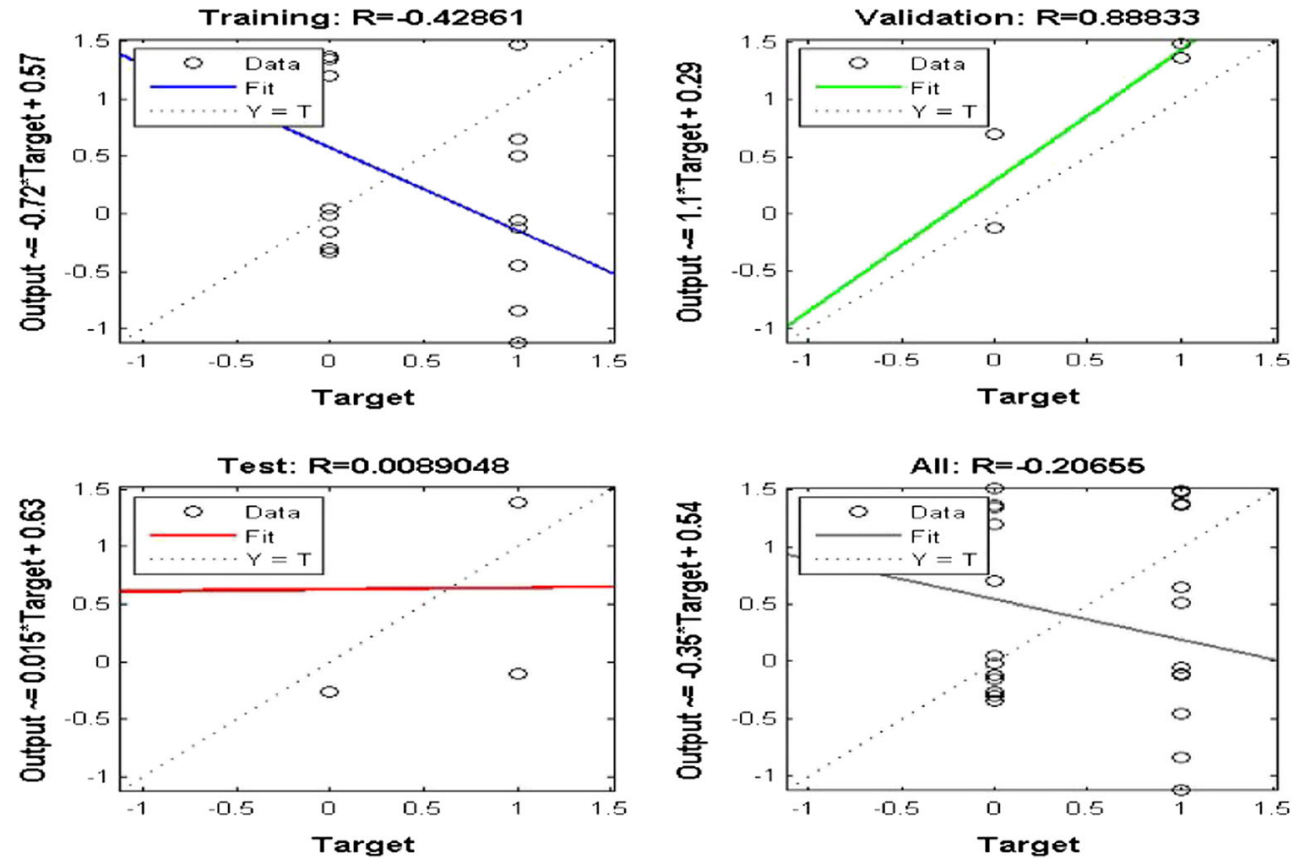

detection of leak appears to the scheme and instantly spread other parts accumulating demand to the contents of line up for pipelines. In MATLAB simulation, ANN toolbox is utilized for designing the network architecture. The ANN architecture with three layers is designed. The layers include the input layer, hidden layer, and the output layer.

\section{Training Neural Network}

Neural network structure consists of three layers-input layer, hidden layer, and output layer. Input layer has one variable, hidden layer $n$ variables, and the output layer consists of three layers. The back propagation algorithm has been applied to train the network with generated data.

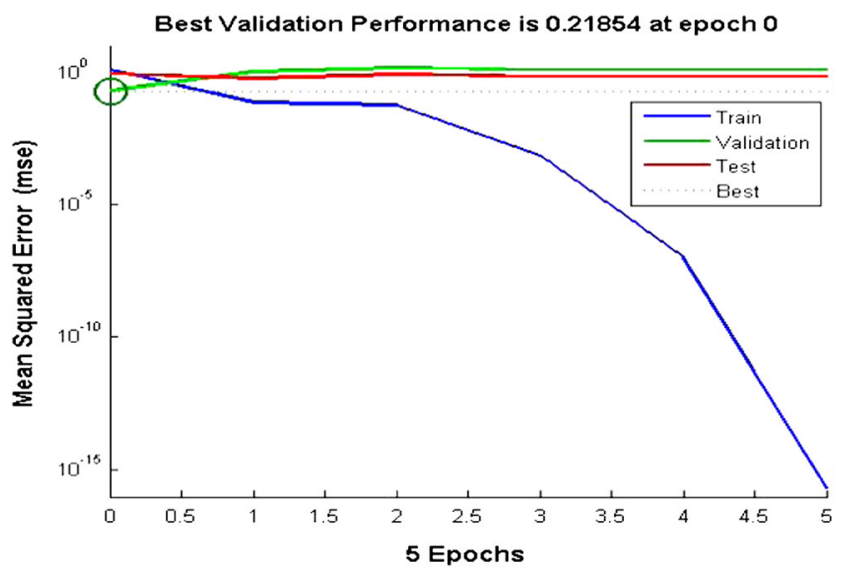

Fig. 4 Proposed ANN validation performance
The network configuration used in proposed technique is mentioned below.

\section{Initial State of the Production Section}

Step 1: Assigning the velocity information for input layers and hidden layers

Initialize the input to the neural network consequently the three input are assigned randomly to represent number of input velocity, release threshold, and capture threshold, respectively.

Step 2: Output calculation for hidden layers

Apply the number of velocity dataset to the input value. Here, $K$ is the input to the network and $k_{1}, k_{2}$, and $k_{3}$ are the output of the forecast value.

$$
\begin{aligned}
& k_{1}=\sum_{r=1}^{n} P_{1} K(r) \\
& k_{2}=\sum_{r=1}^{n} P_{2} K(r) \\
& k_{3}=\sum_{r=1}^{n} P_{3} K(r)
\end{aligned}
$$

Where,

$$
K(r)=\frac{1}{1+\exp \left(-P_{n} \cdot K\right)}
$$


Fig. 5 Error validation testing
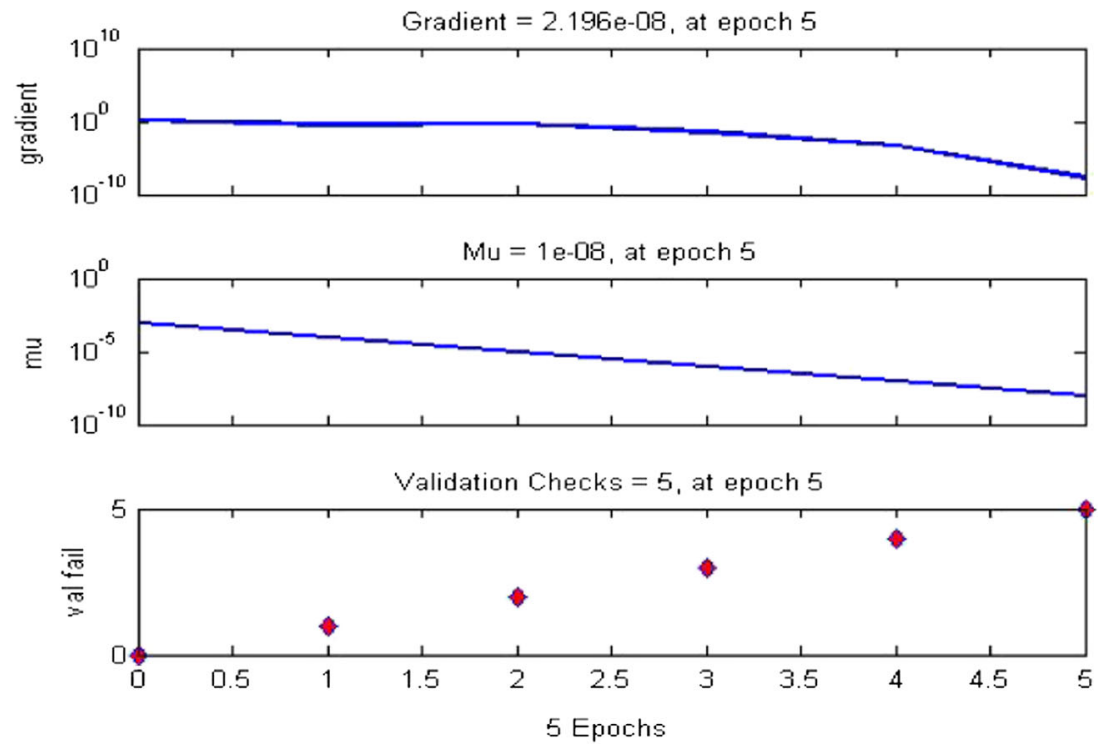

Eqs. (7), (8), (9), and (10) stand for the start function executed at input and output layers orderly.

Step 3: Output calculation for output layers:

$$
\begin{aligned}
& M_{x}=\sum_{i=1}^{D / 2}\left(R_{t} \times C_{t}\right) \\
& M_{y}=\sum_{i=W / 2}^{D}\left(R_{t} \times C_{t}\right) .
\end{aligned}
$$

\section{Release of Parts into the Output Cells}

\section{Step 4: Calculation of errors}

The outcome of step 3 is not an exact production pull control; all designing of systems is based on two stages in series, but the entire thing we present applies $N$ stages in these steps

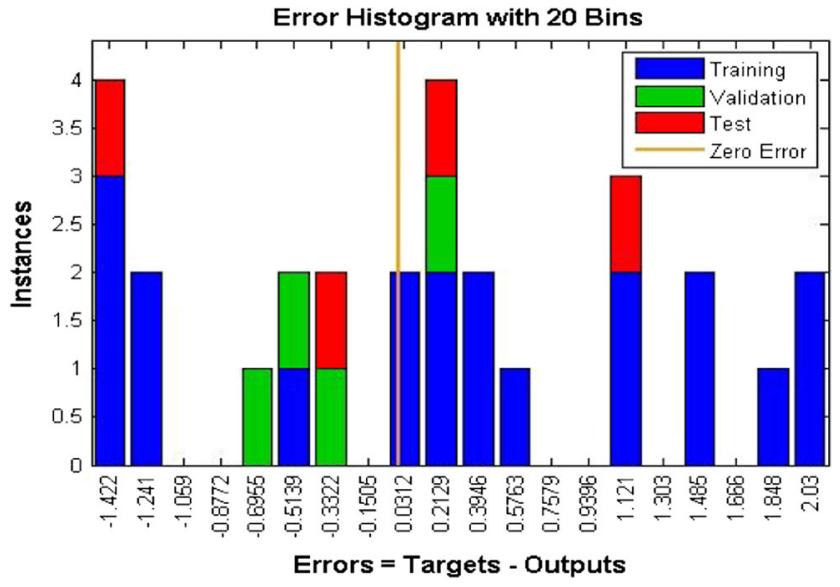

Fig. 6 Error rate of leak detection on pipeline and it has some error value; in material ID, to find the error of the output, get from the step 3 . The error is calculated by the average of difference between starting workstation and outcome workstation is given in the Eqs. (13) and (14).

- Water velocity $A_{i}$ is attached onto $P_{i}$ which is relabeled $\tau(t)$, and together they are transferred downstream to output buffer.

- Demand di is satisfied and is therefore discarded.

$\operatorname{Err}_{x}=\left[\frac{\operatorname{Dis}\left(A_{i}\left(P_{i}\right)\right)-O_{x}}{2}\right]$
$\operatorname{Err}_{y}=\left[\frac{\operatorname{Dis}\left(A_{i}\left(Q_{i}\right)\right)-O_{y}}{2}\right]$

When part $Q_{i}$ terminates processing in $P_{i}$, labeled as $\mathrm{p}_{\mathrm{i}}$, with velocity $o(x, y)$ that was attached onto it, to join $A_{i}$ as a couple.

Step 5: Calculation of back propagation error

$\mathrm{BP}_{\mathrm{Err}}=\frac{\operatorname{Err}_{x}(\mathrm{BP})+\operatorname{Err}_{y}(\mathrm{BP})}{2}$

Step 6: Calculation of weight deviation in hidden layers

$\delta \quad K=B P_{\text {Err }} \times \tau \times l$

Step 7: Calculation of new weights for hidden layers

The new weight for the hidden layers is finding by addition of weight deviation with the old weight of the hidden layer is given in the following Eq. (17).

new $P\left(A_{i}\right)=$ old $\left(A_{i}\right)+\delta \quad K$ 
Step 8: Go to step 2 and find out BP error

After finding the new weights to the hidden layer, subsequently, we need to find the new weights for the input layers since we go to step 2 to find the $\mathrm{BP}_{\mathrm{Err}}$ for new assigned input hidden layer values. This process is repeated until the value of back propagation error become very less. If the value of the back propagation error $\mathrm{BP}_{\mathrm{Err}}$ reached less then, the artificial neural network is considered as well trained.

\section{Simulation Results}

In this research, artificial leaks were built at the pipe and datasets recorded near leak point and analyzed. Pressure differences were recorded by differential pressure transducer (DPT) and analog to digital convertor (ADC) installed for signal conversion. The leakage detection system was comprised of three units: measurement unit, collection unit, and analysis unit.

The pressure transducer is replaced by a loop from feeler in feeler line and a resistor (end-of-line) for detection of leak for hydrocarbon. When the lead is in non-activated state, then the signal screening unit depicts "normal" range. When the lead is absorbed by hydrocarbon, then leak is recognized as transducer reflects resistance to the screening unit. Transducer shows an abrupt rise in resistance if sensing loop is broken. The signal returned to the screening unit and frequency lifted up and monitor recognizes an "open" in feeler (Table 1).

\section{Discussion}

Leakage identification and monitoring in underground hydropipe network is an interesting and challenging work due to lack of easiness of physical monitoring system, and it is mandated to control the depletion using consistent error activity approach of water channel mesh. In this research, we compile online error detection approach of water channel network employing details of pipes such as flow rate (discharge) and pressure. The leak point can be identified as discharge or pressure information's when errors are combined.

In the paper, we used real-time transient method and pressure point analysis for leak detection. The mass, momentum, and energy equations are used in transient method to determine the flow rate. To determine the pipeline leak, we considered difference between the predicted value of the variable and the measured flow. The proposed leak identification approach is based on statistical theory.

In our present work, pressure analysis carried out for leak identification in the pipelines with neural network approach. The presented boundary conditions and parameter update laws into it. In this work, we have focused on replacing the simple model presented in the paper with a modern fluid flow
Table 1 Input and output datasets

\begin{tabular}{|c|c|c|c|}
\hline \multicolumn{2}{|l|}{ Inputs } & \multicolumn{2}{|l|}{ Outputs } \\
\hline Input 1 & Input 2 & Output 1 & Output 2 \\
\hline 0.259 & 0.435 & 1 & 0 \\
\hline 1.245 & 0.410 & 1 & 0 \\
\hline 1.155 & 0.540 & 1 & 0 \\
\hline 0.279 & 0.075 & 0 & 1 \\
\hline 0.279 & 0.075 & 0 & 1 \\
\hline 1.255 & 0.160 & 0 & 1 \\
\hline 1.255 & 0.160 & 0 & 1 \\
\hline 0.239 & 0.245 & 0 & 1 \\
\hline 1.146 & 0.520 & 0 & 1 \\
\hline 1.216 & 0.520 & 0 & 1 \\
\hline 0.458 & 0.075 & 1 & 0 \\
\hline 0.658 & 0.075 & 1 & 0 \\
\hline 0.876 & 0.160 & 1 & 0 \\
\hline 0.950 & 0.160 & 1 & 0 \\
\hline
\end{tabular}

simulator which increased the accuracy of the flow calculations. Therefore, it is expected to improve the leak detection capability described in this paper even further.

\section{Conclusions}

The pressure fluctuations have been studied by neural network approach to detect leakages in pipe. Our proposed method leads an innovative approach for leak detection in pipelines. The weighted average localization algorithm has been introduced to get the precise position of a leak point. The proposed hybrid ANN approach significantly boosts the accuracy in pipeline leak detection process depicted by computational outcomes and minimizes dilemma of complicated decision-making.

In this paper, we mainly focus on the linear pipeline without considering other pipeline cases by ANN approach, and outcomes show its ability and credibility to detect leakages in pipelines. This research is useful since it minimizes loss of energy by increasing prediction accuracy for the leakage in an underground pipe network. In future, more research will be done with different geometry and excellent techniques to enhance the skill of leak detection phenomena.

\section{References}

1. Stoianov I, Nachman L, Madden S, Tokmouline T, Csail M (2007) PIPENET: a wireless sensor network for pipeline monitoring, 6th international symposium on information processing in sensor networks, PRO, pp.264-273 
2. Simpson A, Dandy G, Murphy L (1994) Genetic algorithms compared to other techniques for pipe optimization. J Water Resour Plann Manag 120(4):423-443

3. Batziasa FA, Siontoroua CG, Spanidis PMP (2011) Designing a reliable leak bio-detection system for natural gas pipelines. $\mathrm{J}$ Hazard Mater 186(1):35-58

4. Shyam S, Constantinides K, Phadke S, Bertacco V, Austin T (2006) Ultra low-cost defect protection for microprocessor pipelines. In proceedings of the 12th international conference on Architectural support for programming languages and operating systems, ACM, New York pp. 73-82

5. Yoon S, Ye W, Heidemann J, Littlefield B, Shahabi C (2011) SWATS: wireless sensor networks for steam flood and water flood pipeline monitoring. IEEE Netw 25(1):50-56

6. Wagner J, Shamir U, Marks D (1988) Water distribution reliability: simulation methods. J Water Resour Plan Manag 114(3):276-294

7. HE Emara-Shabaik, YA Khulief and Hussaini I, Proc Inst Mech Eng Part I: J Syst Control Eng, Vol. 216, No. 6, pp. 497-512, 2002

8. Sægrov S, MeloBaptista JF, Conroy P, Herz RK, Gauffre PL, Mossc G, Oddevald JE, Rajanig B, Schiattih M (1999) Rehabilitation of water networks: survey of research needs and on-going efforts. Urban Water 1(1):15-22

9. Kroll A, Baetz W, Peretzki D (2009) On autonomous detection of pressured air and gas leaks using passive IR-thermography for mobile robot application, IEEE International Conference on Robotics and Automation, pp.921-926

10. Kim J-H, Sharma G, Boudriga N, Iyengar SS (2010) SPAMMS: a sensor-based pipeline autonomous monitoring and maintenance system, In preceding of communication systems and networks (COMSNETS), pp. 1-10

11. Jawhar I, Mohamed N, Shuaib K (2007) A framework for pipeline infrastructure monitoring using wireless sensor networks, Wireless Telecommunications Symposium, pp. 1-7

12. Covas D, Ramos H, de Almeida A (2005) Standing wave difference method for leak detection in pipeline systems. J Hydraul Eng 131(12):1106-1116

13. Estes A, Frangopol D (1999) Repair optimization of highway bridges using system reliability approach. J Struct Eng 125(7):766-775

14. Beck S, Curren M, Sims N, Stanway R (2005) Pipeline network features and leak detection by cross-correlation analysis of reflected waves. J Hydraul Eng 131(8):715-723
15. Vítkovský J, Lambert M, Simpson A, Liggett J (2007) Experimental observation and analysis of inverse transients for pipeline leak detection. J Water Resour Plan Manag 133(6):519 530

16. Sun Z, Wang P, Vuran MC, Al-Rodhaan MA, Al-Dhelaan AM, Akyildiz IF (2011) MISE-PIPE: magnetic induction-based wireless sensor networksfor underground pipeline monitoring. Ad Hoc Netw 9:218-227

17. Colombo AF, Lee P, Karney BW (2009) A selective literature review of transient-based leak detection methods. J Hydro Environ Res 2:212-227

18. Abhulimen KE, Susu AA (2004) Liquid pipeline leak detection system: model development and numerical simulation. Chem Eng J 97:47-67

19. MingLiu SZ, Azhou D (2005) Fast leak detection and location of gas pipelines based on an adaptive particle filter. Int J Appl Math Comput Sci 15(4):541-550

20. Taghvaei M, Beck SBM, Staszewsk WJ (2006) Leak detection in pipelines using cepstrum analysis. Meas Sci Technol 17:367-372

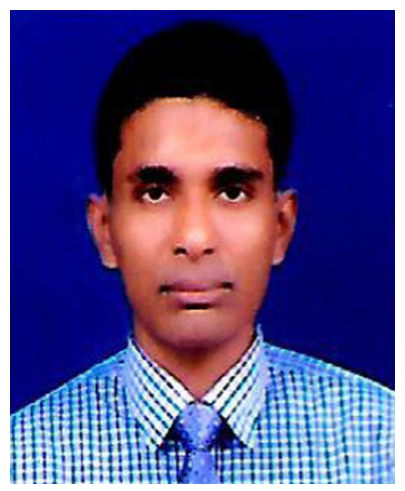

Mr. Uttam Roy completed Bachelor of Mechanical Engineering from Jadavpur University, Kolkata in the year 2003 and Master of Engineering from Bengal Engineering and Science University, Shibpur in the year 2005. He is a researcher at National Institute of Technology, Agartala.

He has more than twelve years of professional experience in reputed government and private organizations. He published more than ten papers in reputed international journals and two international books/monographs. He has filed a patent at US patent house. He is also associated with industry projects as an Engineer in R\&D section. 